\title{
铁催化硬脂酸选择性氢化制硬脂醇
}

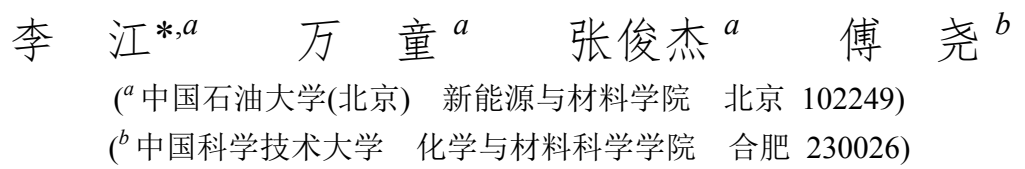

\begin{abstract}
摘要 长链脂肪酸是一种重要的生物质原料, 其氢化制备的脂肪醇由于用途广泛以及市场价值不断增长, 近年来引起 了人们的广泛关注. 报道了一例使用非均相铁催化硬脂酸选择性加氢制备硬脂醇的实例. 与其他非贵金属元素(如钴和 镍)相比, 铁在地壳中的丰度高出 $3000 \sim 30000$ 倍, 价格仅为 $20 \sim 150$ 分之一, 因此该方法更经济, 更有吸引力. 催化剂 是通过将铁和氮掺杂碳前驱体同时热解到氧化铝载体上制备的. 制备催化剂时最佳热解温度为 $900{ }^{\circ} \mathrm{C}$, 最佳铁的质量 分数为 $20 \%$. 反应最佳反应温度为 $320{ }^{\circ} \mathrm{C}$, 最佳氢气压力为 $4 \mathrm{MPa}$. 产物随时间分布曲线表明硬脂酸在 $0.5 \mathrm{~h}$ 内迅速转 化为硬脂醇, 收率为 $88.6 \% .4 \mathrm{~h}$ 后逐渐转化为十八烷，收率为 $90 \%$.
\end{abstract}

关键词 生物质转化; 氢化; 铁; 脂肪酸; 脂肪醇

\section{Iron-Catalyzed Selective Hydrogenation of Stearic Acid to Stearyl Alcohol}

\author{
Li, Jiang ${ }^{*, a} \quad$ Wan, Tong $^{a} \quad$ Zhang, Junjie $^{a} \quad \mathrm{Fu}, \mathrm{Yao}^{b}$ \\ ( ${ }^{a}$ College of New Energy and Materials, China University of Petroleum, Beijing 102249) \\ ( ${ }^{b}$ School of Chemistry and Materials Science, University of Science and Technology of China, Hefei 230026)
}

\begin{abstract}
The utilization of sustainable resources such as biomass to produce fuels and chemicals has recently attracted significant attention due to the depletion of fossil reserves, increasing energy demand, and growing environmental concerns. Long-chain fatty acids, which are major constituents of plant oil, are important feedstock for biorefinery. Besides producing well-known biodiesels, the hydrogenation of fatty acids to fatty alcohols has recently drawn significant attention due to the versatility and growing market value of fatty alcohols. An example of heterogenous iron-catalyzed selective hydrogenation of stearic acid to stearyl alcohol is reported. Comparing with other reported non-noble metal centers, such as $\mathrm{Co}$ and $\mathrm{Ni}$, $\mathrm{Fe}$ is $3000 \sim 30000$ times more abundant and 20 150 times cheaper, thus making our method more economic and attractive. The iron catalyst was prepared by simultaneous pyrolysis of iron precursor $\left[\mathrm{Fe}(\mathrm{acac})_{3}\right]$ and nitrogen-doped carbon precursor (melamine) onto alumina, bearing $\mathrm{Fe}_{3} \mathrm{C}$ active phase and nitrogen-doped carbon-alumina hybrid support. The optimization of preparation parameter showed that the optimal pyrolysis temperature is $900{ }^{\circ} \mathrm{C}$, while the best mass fraction of iron is $20 \%$. The replacement of $\mathrm{Fe}(\mathrm{acac})_{3}$ with $\mathrm{Fe}\left(\mathrm{NO}_{3}\right)_{3}$ led to inferior catalytic performance, which was due to undesired redox reaction between $\mathrm{NO}_{3}^{-}$and melamine during pyrolysis that hampered the reaction between $\mathrm{Fe}$ and melamine to form $\mathrm{Fe}_{3} \mathrm{C}$ active phase. Instead, hercynite phase became the predominant phase. The exploration of reaction parameter showed that the optimal reaction temperature is $320{ }^{\circ} \mathrm{C}$, and the best $\mathrm{H}_{2}$ pressure is $4 \mathrm{MPa}$. The time course for stearic acid conversion shows that stearic acid was rapidly converted into stearyl alcohol with yield of $88.6 \%$ within $0.5 \mathrm{~h}$, and then gradually converted into octadecane with yield of $90 \%$ at $4 \mathrm{~h}$. The unsatisfactory stability of the iron catalyst is probably due to the decomposition of $\mathrm{Fe}_{3} \mathrm{C}$ active phase to metallic Fe phase during recycling tests.
\end{abstract}

Keywords biomass conversion; hydrogenation; iron; fatty acid; fatty alcohol

生物质作为唯一含有有机碳的可再生资源, 其取代 化石能源生产液体燃料及化学品受到了研究人员的广
泛关注 ${ }^{[1 ~ 3]}$. 由三分子脂肪酸和一分子甘油经酯化作用 形成的油脂类化合物, 是制备生物液体燃料的一种重要

\footnotetext{
* Corresponding author. E-mail: 1ijiang@cup.edu.cn

Received February 26, 2019; revised May 13, 2019; published online July 2, 2019.

Project supported by the National Natural Science Foundation of China (No. 21702227) and the Science Foundation of China University of Petroleum (Beijing) (No. 2462014YJRC037).

国家自然科学基金(No. 21702227)和中国石油大学(北京)校启动基金(No. 2462014YJRC037)资助项目.
} 
原料 ${ }^{[4]}$. 除制备生物柴油外, 脂肪酸还可选择性氢化制 备脂肪醇. 作为一类重要的高附加值化学品, 它们可用 于制备润滑油、树脂、香水和化妆品等多种产品 ${ }^{[5]}$. 近 年来, 全球脂肪醇的市场规模已经达到了 19 亿美元, 年 增长率为 $7.3 \%$, 且未来市场规模仍有很大的提升空 间 ${ }^{[6]}$.

在前期的研究中, 铜铬催化剂已被用于脂肪酸选择 性氢化制脂肪醇反应中. 在 $250{ }^{\circ} \mathrm{C}$ 的反应温度, $22 \mathrm{MPa}$ 的反应压力下, 7 种羧酸被转化为相应的醇, 产率为 $80 \% \sim 98 \%{ }^{[7]}$. 由于 $\mathrm{Cr}$ 的使用有可能造成严重的环境问 题, 近些年来新的催化体系已被不断发展出来. 相比于 均相催化体系, 非均相催化过程具有易与产物分离、体 系容易放大及制备成本较低等优势, 备受研究人员青

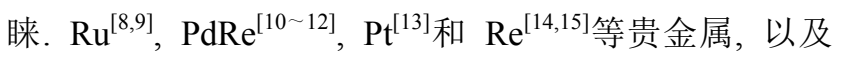
$\mathrm{Cu}^{[16,17]}, \mathrm{Ni}^{[18 \sim 20]}$ 和 $\mathrm{Co}^{[21,22]}$ 等非贵金属，均已被报道可以 高选择性催化该氢化反应. 例如, 氧化钛负载的 RuSn 催化剂 $(\mathrm{Ru} / \mathrm{Sn}$ 物质的量之比约为 $1: 2)$ 在 $250{ }^{\circ} \mathrm{C}$ 的反应 温度下可以选择性将油酸转化为对应的不饱和醇 ${ }^{[9]}$. 值 得注意的是, 氧化钛负载的 Pt 催化剂可以在低至 $130{ }^{\circ} \mathrm{C}$ 的反应温度下将硬脂酸转化为硬脂醇, 反应时氢 气压力为 $2 \mathrm{MPa}^{[13]}$. 对于非贵金属催化剂, 尽管 $\mathrm{Fe}$ 复合 的 $\mathrm{Cu}, \mathrm{Ni}$ 催化剂可以以 $>90 \%$ 的选择性将硬脂酸转化为 硬脂醇 ${ }^{[16,20]}$, 但由于 $\mathrm{Fe}$ 本身较低的反应活性, 利用单金 属 $\mathrm{Fe}$ 实现脂肪酸氢化制醇尚未有前人研究报道.

铁是一种地壳含量丰富、生态友好、相对无毒且廉 价的元素, 非常适合作为生物质转化过程中贵金属催化 剂的替代物. 与 $\mathrm{Co}, \mathrm{Ni}, \mathrm{Mo}$ 和 $\mathrm{W}$ 等非贵金属相比, 铁在 地壳的丰度要高 $3000 \sim 30000$ 倍, 价格要便宜 $20 \sim 150$ 倍 ${ }^{[23]}$. 但是铁的低活性极大程度上限制了其在生物质 转化过程的应用. 近期, 利用氮掺杂提高铁催化剂的活 性引起了研究人员的极大关注. 2013 年, Beller 课题组 ${ }^{[24]}$ 发现将醋酸亚铁和 1,10-菲罗啉在活性炭上同时热解制 备的 Fe-phen-C 催化剂可以高效地催化硝基芳烃还原. 进一步研究表明该催化剂对于 $\mathrm{N}$-杂环氧化脱氢和无氢 还原胺化同样具有良好的催化效果 ${ }^{[25,26]}$. 受他们工作的 启发, 我们将 Fe-N-C 催化剂成功地运用到糠醛转移氢 化 ${ }^{[27]} 、 5$-着甲基糠醛选择性加氢脱氧 ${ }^{[28,29]}$ 和木质素 $\mathrm{C}$ $\mathrm{O}$ 键选择性裂解 ${ }^{[30]}$ 等生物质转化反应中. 最近, 我们发 现氮杂碳及氧化铝复合物负载的铁催化剂可以高选择 性地催化转化羧酸化合物制备相对应的烃 ${ }^{[23]}$. 催化剂 中的 $\mathrm{Fe}_{3} \mathrm{C}$ 活性相对羧酸氢化制醇步骤起了决定作用, 而氮杂碳及氧化铝复合物结构则对醇加氢脱氧制烃步 骤至关重要. 在这一工作基础上, 我们首次实现非均相 铁催化硬脂酸高选择性加氢制备相应的脂肪醇, 铁金属 中心及复合物载体的存在对催化剂的加氢活性至关重
要. 通过对催化剂制备及反应参数进行调控, 我们发现 在 $320{ }^{\circ} \mathrm{C}$ 的反应温度下反应 $0.5 \mathrm{~h}$, 即可将硬脂酸以 $88.6 \%$ 的选择性氢化为硬脂醇. 这一结果为硬脂酸选择 性氢化制醇提供了一种新的具有经济性的铁基催化体 系.

\section{1 结果与讨论}

\section{1 催化剂篮选}

首先对反应的催化剂进行了笁选(表 1). 空白反应 的结果表明反应无法在无催化剂时进行. 当使用 $20 \%$ $\mathrm{Fe}-\mathrm{N}-\mathrm{C} @ \mathrm{Al}_{2} \mathrm{O}_{3}-900$ (铁的质量分数为 $20 \%$ ) 催化剂时, 反 应 $0.5 \mathrm{~h}$ 时醇的收率可达到 $88.6 \%$ (Entry 2). 经过文献调 研，这是首例使用单金属铁实现脂肪酸高选择性转化制 备相应的脂肪醇. 对于催化剂详尽的表征参见近期研究 工作 ${ }^{[23]}$. 无铁负载及氮掺杂的催化剂均表现出非常差 的反应活性(Entries 3 5), 证明催化剂中铁的负载以及 氮的掺杂均非常重要. 对催化剂热解温度的调节表明 $900{ }^{\circ} \mathrm{C}$ 是最优的热解温度(Entries $6 \sim 8$ ). $\mathrm{X}$ 射线(XRD) 表征显示仅在 $900{ }^{\circ} \mathrm{C}$ 的热解温度下存在明显的 $\mathrm{Fe}_{3} \mathrm{C}$ 相 的衍射峰(图 1), 证明 $\mathrm{Fe}_{3} \mathrm{C}$ 相对于羧酸加氢制醇至关重 要, 这也与我们的前期研究结论一致. 使用扫描电镜 (SEM) 表征 Fe-N-C@ $\mathrm{Al}_{2} \mathrm{O}_{3}-900$ 催化剂的形貌, 可以观 察到明显的氧化铝片层结构(图 1). 其他载体如 $\mathrm{C}, \mathrm{TiO}_{2}$ 及 $\mathrm{SiO}_{2}$ 负载的铁催化剂均对该氢化反应表现出非常差 的活性(Entries 9 11), 醇的产率 $<5 \%$. 当铁源由乙酰 丙酮铁变为硝酸铁时，醇的产率降低到了 $10.8 \%$ (Entries 12). 这是由于硝酸根离子在热解过程中可能与氮 源发生了氧化还原反应，导致铁未在热解过程中形成碳 化铁相, 而是与载体发生反应形成了尖晶石相(图 1). 这 种尖晶石相同样可以在 $\mathrm{Fe}-\mathrm{Al}_{2} \mathrm{O}_{3}-900$ 催化剂的 XRD 谱 图中观察到(图 1). 这些催化剂非常差的反应活性表明 尖晶石相的形成不利于氢化反应的发生. 将氮源替换为 前期合成 Fe-N-C 催化剂常用的 1,10-菲罗啉后, 醇的产 率降低至 58.8\%(Entries 13). 这可能是因为 1,10-菲罗啉 的氮含量远比三聚氧胺低, 为了保证催化剂前体中具有 同样的 $\mathrm{N}$ 含量, 需要大大增加 1,10-菲罗啉的加入量, 从 而导致氧化铝载体的结构发生了改变(图 1). 铁负载量 的研究表明最优的负载量的质量分数为 $20 \%$ (Entries 14 17). 使用 Fe L 边 X 射线吸收精细结构(Fe L-edge XANES)对不同铁负载量的催化剂进行表征发现质量分 数为 $10 \%$ 的铁负载量的催化剂在 $\mathrm{L} 3$ 区域 $\left(2 \mathrm{p}_{3 / 2} \rightarrow 3 \mathrm{~d}\right.$ 转 变)有明显的 $\mathrm{Fe}^{0}$ 峰 $\left(709 \mathrm{eV}\right.$, 图 2) ${ }^{[31]}$. 相比于对照样氧化 铁, 铁催化剂中 $\mathrm{Fe}^{3+}$ 的特征峰随着负载量的降低逐渐向 低场移动, 证明 $\mathrm{Fe}$ 的价态不断降低, 间接表明催化剂的 氧空位不断增加, 这对加氢反应是有利的. 因此, 结合 
表 1 催化剂笁选 ${ }^{a}$

Table 1 Catalyst screening

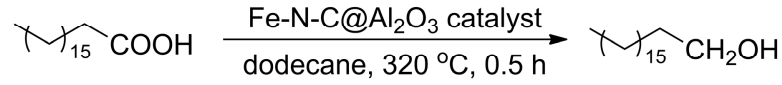

\begin{tabular}{|c|c|c|c|c|c|c|c|}
\hline \multirow{2}{*}{ Entry } & \multirow{2}{*}{ Catalyst } & \multirow{2}{*}{ Conv./\% } & \multicolumn{4}{|c|}{ Yield $^{b} / \%$} & \multirow{2}{*}{ Carbon balance } \\
\hline & & & $\mathrm{C} 18-\mathrm{OH}$ & $\mathrm{C} 17$ & $\mathrm{C} 18$ & $\mathrm{C} 17-\mathrm{CHO}$ & \\
\hline 1 & - & 33.3 & 0.5 & 0.5 & 0.1 & 0.2 & 3.9 \\
\hline 2 & 20\% Fe-N-C@ $@ \mathrm{Al}_{2} \mathrm{O}_{3}-900$ & $>99$ & 88.6 & 1.1 & 2.4 & 0.4 & 92.5 \\
\hline 3 & $20 \% \mathrm{Fe}-\mathrm{Al}_{2} \mathrm{O}_{3}-900$ & 21.6 & 0.9 & 0.9 & 0.1 & 2.2 & 19.0 \\
\hline 4 & $\mathrm{~N}-\mathrm{C} @ \mathrm{Al}_{2} \mathrm{O}_{3}-900$ & 42.3 & 0.5 & 0.5 & 0.4 & 0.4 & 4.3 \\
\hline 5 & $\mathrm{Al}_{2} \mathrm{O}_{3}$ & 43.3 & 7.9 & 1.0 & 0.9 & 4.1 & 32.1 \\
\hline 6 & $20 \%$ Fe-N-C@ $@ \mathrm{Al}_{2} \mathrm{O}_{3}-550$ & 98.3 & 80.4 & 1.5 & 14.3 & 0 & 97.9 \\
\hline 7 & 20\% Fe-N-C@ $\mathrm{Al}_{2} \mathrm{O}_{3}-700$ & 95.7 & 34.1 & 0.7 & 3.8 & 3.0 & 43.5 \\
\hline 8 & $20 \% \mathrm{Fe}-\mathrm{N}-\mathrm{C} @ \mathrm{Al}_{2} \mathrm{O}_{3}-1100$ & 97.1 & 47.9 & 1.6 & 11.8 & 2.6 & 65.8 \\
\hline 9 & 20\% Fe-N-C@ $@ \mathrm{SiO}_{2}-900$ & 88.5 & 1.0 & 1.3 & 0.1 & 1.1 & 4.0 \\
\hline 10 & $20 \%$ Fe-N-C-900 & 92.1 & 4.3 & 1.6 & 0.7 & 1.8 & 9.1 \\
\hline 11 & 20\% Fe-N-C@ $@ \mathrm{TiO}_{2}-900$ & 66.9 & 3.6 & 0.9 & 0.4 & 3.0 & 11.8 \\
\hline 12 & $20 \% \mathrm{Fe}\left(\mathrm{NO}_{3}\right)_{3}-\mathrm{N}-\mathrm{C} @ \mathrm{Al}_{2} \mathrm{O}_{3}-900$ & 80.6 & 10.8 & 1.2 & 0.6 & 2.2 & 18.4 \\
\hline 13 & 20\% Fe-phen-C@ $@ \mathrm{Al}_{2} \mathrm{O}_{3}-900$ & 96.2 & 58.8 & 0.9 & 4.4 & 3.5 & 70.3 \\
\hline 14 & 3\% Fe-N-C@ $\mathrm{Al}_{2} \mathrm{O}_{3}-900$ & 89.4 & 52.6 & 1.0 & 1.1 & 2.9 & 64.4 \\
\hline 15 & 5\% Fe-N-C@ $\mathrm{Al}_{2} \mathrm{O}_{3}-900$ & 94.7 & 21.6 & 0.6 & 0.8 & 3.9 & 28.4 \\
\hline 16 & $10 \% \mathrm{Fe}-\mathrm{N}-\mathrm{C} @ \mathrm{Al}_{2} \mathrm{O}_{3}-900$ & 98.0 & 62.0 & 0.6 & 0.6 & 1.8 & 66.3 \\
\hline 17 & $30 \% \mathrm{Fe}-\mathrm{N}-\mathrm{C} @ \mathrm{Al}_{2} \mathrm{O}_{3}-900$ & 99.3 & 46.0 & 0.8 & 1.5 & 2.2 & 50.9 \\
\hline
\end{tabular}

${ }^{a}$ Reaction conditions: $0.5 \mathrm{mmol}$ of stearic acid, $20 \mathrm{~mL}$ of solvent, $0.1 \mathrm{~g}$ of catalyst, $4 \mathrm{MPa} \mathrm{H}_{2}, 320{ }^{\circ} \mathrm{C}$, and $0.5 \mathrm{~h} .{ }^{b} \mathrm{C} 18-\mathrm{OH}$ refers to octadecanol, $\mathrm{C} 17 \mathrm{refers}$ to heptadecane, $\mathrm{C} 18$ refers to octadecane, and $\mathrm{C} 17-\mathrm{CHO}$ refers to octadecanal.
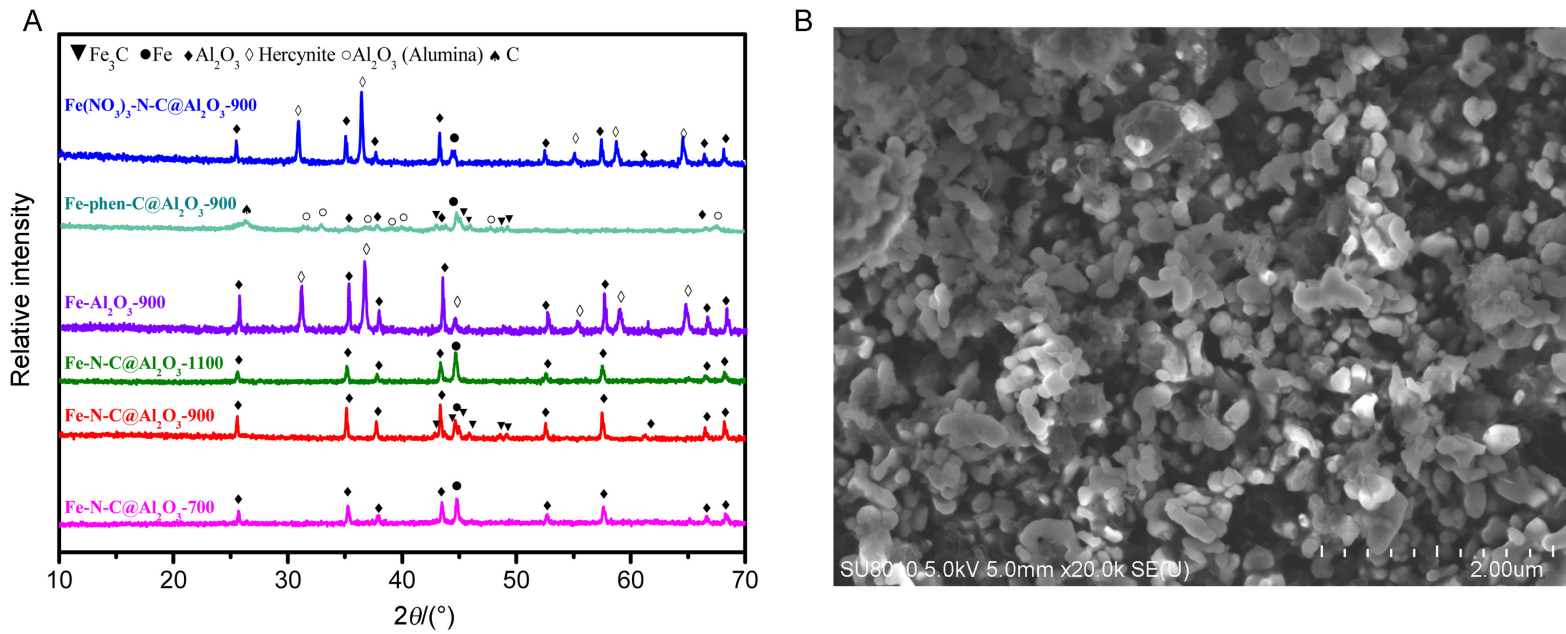

图 1 不同条件下制备的 $\mathrm{Fe}-\mathrm{N}-\mathrm{C} @ \mathrm{Al}_{2} \mathrm{O}_{3}$ 和 $\mathrm{Fe}-\mathrm{Al}_{2} \mathrm{O}_{3}-900$ 催化剂的 XRD 谱图(A)和 $\mathrm{Fe}-\mathrm{N}-\mathrm{C} @ \mathrm{Al}_{2} \mathrm{O}_{3}-900$ 催化剂的 SEM 谱图(B)

Figure 1 XRD patterns of $\mathrm{Fe}-\mathrm{N}-\mathrm{C} @ \mathrm{Al}_{2} \mathrm{O}_{3}$ catalyst prepared under different conditions and $\mathrm{Fe}-\mathrm{Al}_{2} \mathrm{O}_{3}-900$ catalyst (A), and SEM image of $\mathrm{Fe}-\mathrm{N}-\mathrm{C} @ \mathrm{Al}_{2} \mathrm{O}_{3}-900$ catalyst (B)

$\mathrm{X}$ 射线衍射(XRD)和 X 射线吸收近边结构(XANES)的表 征我们推断催化剂对硬脂酸加氢的活性与 $\mathrm{Fe}_{3} \mathrm{C}$ 活性相 及氧空位的存在非常相关.

\section{2 温度效应}

在篮选出最优的反应催化剂后, 对反应参数进行了 研究. 如图 3 所示, 反应温度对铁催化羧酸氢化制醇反 应有着很大影响. 在较低的反应温度下如 260 和 $280{ }^{\circ} \mathrm{C}$ 反应时, 不论是主产物硬脂醇还是副产物烷烃和酫的产
率都很低. 当反应温度提高到 $300{ }^{\circ} \mathrm{C}$ 时, 硬脂醇的产率 提高到了 $61.2 \%$ ，同时以 $15.5 \%$ 的产率生成了硬脂醛. 前人的文献表明对于多种催化剂, 硬脂酸的转化率和硬 脂醇的产率在一定的温度区间内都会有不同程度的突 然提升现象 ${ }^{[16,20,21]}$. 我们推测, 在本工作中, 此现象可 能是因为随着反应温度的提高, 反应物分子更加容易进 入到催化剂的氧空位中. 当温度从 $280{ }^{\circ} \mathrm{C}$ 升到 $300{ }^{\circ} \mathrm{C}$ 时, 大量的反应物分子进入了催化剂的氧空位，从而大 


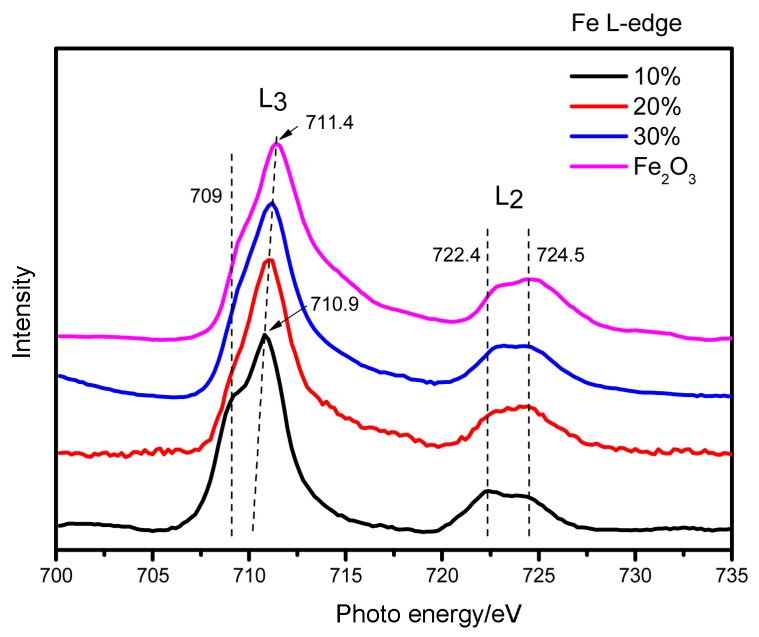

图 2 不同铁含量的 Fe-N-C@ $\mathrm{Al}_{2} \mathrm{O}_{3}$ 催化剂和对照样 $\mathrm{Fe}_{2} \mathrm{O}_{3}$ 的 Fe L 边 XANES 谱图

Figure 2 Fe L-edge XANES spectra of Fe-N-C@ $\mathrm{Al}_{2} \mathrm{O}_{3}-900$ catalysts with different mass fraction of $\mathrm{Fe}$ and control sample $\mathrm{Fe}_{2} \mathrm{O}_{3}$

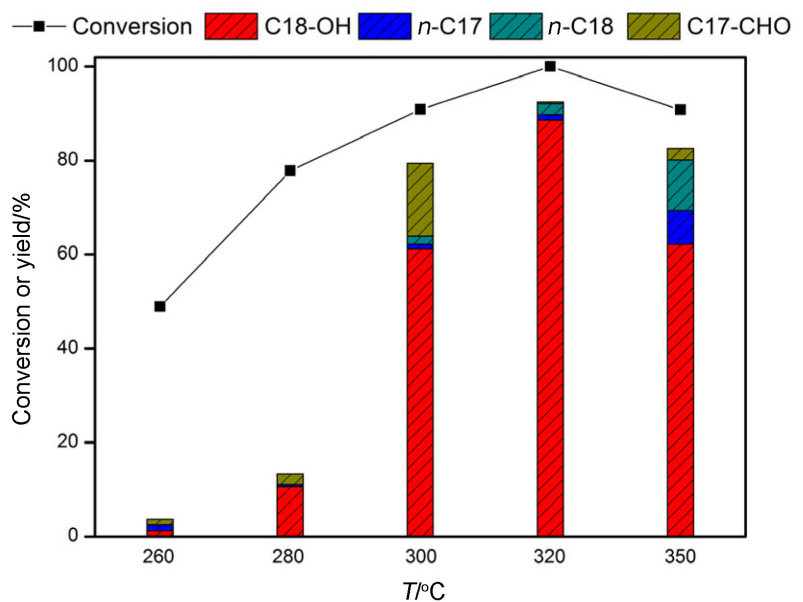

图 3 反应温度对于铁催化硬脂酸氢化制硬脂醇的影响

Figure 3 Effect of reaction temperature on iron-catalyzed hydrogenation of stearic acid to stearyl alcohol

大加速了反应, 使得硬脂酸的转化率和硬脂醇的产率大 幅度提高. 大量的硬脂醛副产物的生成表明硬脂醛极有 可能是硬脂酸加氢制备硬脂醇过程的中间体. 反应温度 进一步提升至 $320{ }^{\circ} \mathrm{C}$ 时, 硬脂醇的产率达到了最大值 (88.6\%). 继续提升反应温度到 $350{ }^{\circ} \mathrm{C}$ 会使硬脂酸转化 率下降, 烷烃副产物的产率增加. 因此, 后续反应参数 探索将在 $320{ }^{\circ} \mathrm{C}$ 的反应温度下进行.

\section{3 氢压效应}

在研究了温度效应后, 同样研究了氢气压力对于反 应的影响. 如图 4 所示, 在 $320{ }^{\circ} \mathrm{C}$ 的反应温度下, 当氢 压为 $1 \mathrm{MPa}$ 时, 硬脂酸的转化率为 $17.1 \%$, 而硬脂醇的 产率达到 $11.8 \%$. 反应压力提升到 $2 \mathrm{MPa}$ 时, 硬脂酸的
转化率提高到了 $78.9 \%$, 硬脂醇和十八烷的产率分别提 高到了 $41 \%$ 和 $9.6 \%$. 继续提高氢气压力至 $4 \mathrm{MPa}$ 时硬脂 醇的产率达到了最大值 $88.6 \%$. 然而, 将氢气压力再提 高至 $6 \mathrm{MPa}$ 会导致十八烷的产率提高到 $16.6 \%$. 相应的, 硬脂醇产率下降到了 $72.3 \%$, 说明在高的氢气压力下, 醇容易继续发生氢解反应生成十八烷. 因此, 为了使反 应停留在硬脂醇阶段继而提高硬脂醇的选择性，后续反 应优化将在 $4 \mathrm{MPa}$ 的反应压力下进行.

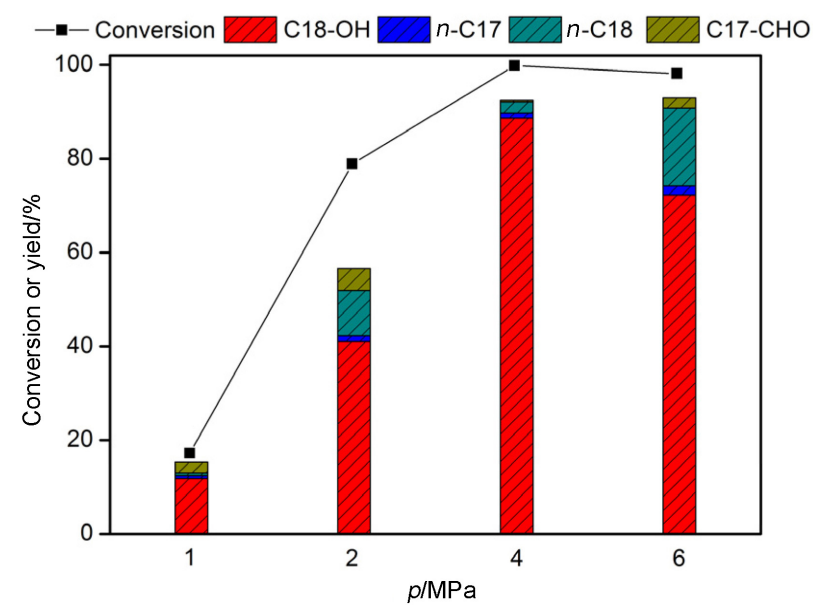

图 4 反应氢压对于铁催化硬脂酸氢化制硬脂醇的影响 Figure 4 Effect of $\mathrm{H}_{2}$ pressure on iron-catalyzed hydrogenation of stearic acid to stearyl alcohol

\section{4 时间曲线}

为了更好地研究非均相铁催化硬脂酸转化过程的 反应路径和中间体，制作了反应过程中产物随时间的分 布曲线(图 5). 反应进行 $5 \mathrm{~min}$ 后, 硬脂酸转化率达到了 $12 \%$, 硬脂醇的产率为 $5.6 \%$, 而硬脂醛的产率为 $2.3 \%$. 反应 $10 \mathrm{~min}$ 后，硬脂酸转化率达到了 $50.7 \%$, 硬脂醇的 产率提高到了 $38.7 \%$. 反应 $30 \mathrm{~min}$ 后，硬脂酸基本转化 完毕，硬脂醇的产率达到了最大值 $88.6 \%$. 随着反应的 继续进行，硬脂醇的产率不断下降，相应的十八烷的产

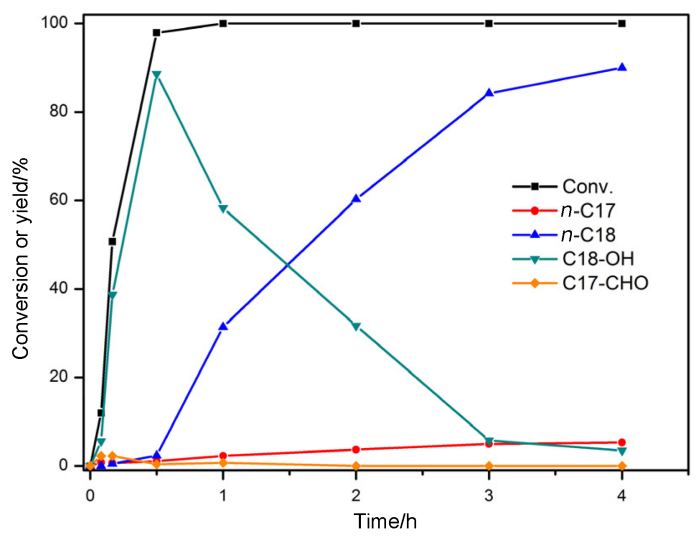

图 5 铁催化硬脂酸转化过程时间曲线

Figure 5 Time course for iron-catalyzed conversion of stearic acid 
率则不断上升，最终在反应 $4 \mathrm{~h}$ 后达到 $90 \%$ 的产率，而 此时硬脂醇的产率仅为 $3.5 \%$. 对样品进行气质分析后 发现反应过程中有痕量的十八烯生成, 表明十八烷很有 可能是硬脂醇经脱水再氢化过程生成的. 结合前文反应 参数的考察结果, 铁催化硬脂酸转化过程的反应路径如 图 6 所示.

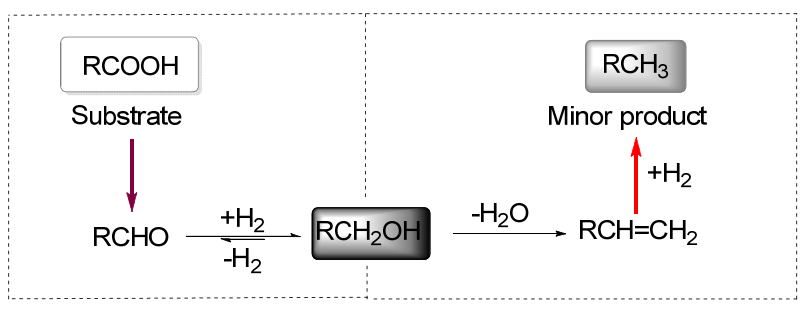

Step 1: Hydrogenation

Step 2: HDO

图 6 铁催化硬脂酸转化过程可能的反应路径

Figure 6 Proposed reaction pathway for iron-catalyzed conversion of stearic acid

\section{5 稳定性实验}

最后, 对催化剂的稳定性进行了考察. 反应结束后, 催化剂通过离心的方式与反应溶液分离, 随后用溶剂洗 涤几次后直接用于下次反应. 如图 7a 所示, 随着催化剂 的重复利用次数的增加, 无论是硬脂酸的转化率还是硬 脂醇的产率都会显著下降. $\mathrm{Fe}_{3} \mathrm{C}$ 相是羧酸加氢制醇的关 键活性位点, 而在重复使用过程中 $\mathrm{Fe}_{3} \mathrm{C}$ 相会降解为单 质铁相 (图 7b), 催化剂的加氢活性随之降低. 进一步的 工作将致力于合成具有高稳定性 $\mathrm{Fe}_{3} \mathrm{C}$ 相的非均相铁催 化剂.

\section{3 结论}

由于脂肪醇的高附加值以及其巨大的市场容量, 开 发高效经济的催化体系用于脂肪酸加氢生产脂肪醇非 常重要. 报道了首例非均相铁催化转化硬脂酸制备硬脂 醇的实例. $\mathrm{Fe}_{3} \mathrm{C}$ 活性相、氮杂碳-氧化铝的复合结构及氧 空位的存在对于该氢化过程非常重要. 通过对催化剂制 备条件及反应因素的优化, 硬脂醇的最高产率达到了 $88.6 \%$. 该工作为硬脂酸选择性氢化制硬脂醇提供了一 条新的具有经济性的催化路线. 进一步的工作将研究如 何制备具有高稳定性 $\mathrm{Fe}_{3} \mathrm{C}$ 相的铁催化剂.

\section{4 实验部分}

\section{1 实验材料}

乙酰丙酮铁( $98 \%)$ 和金属氧化物载体如 $\mathrm{Al}_{2} \mathrm{O}_{3}$ (30 $\mathrm{nm}$, 亲水性, $99.9 \%)$ 、二氧化钛 $(99 \%)$ 、二氧化硅 $(99.9 \%)$ 及十二烷 $(98 \%)$ 购自阿拉丁试剂有限公司. 九水合硝酸 铁(98.5\%)和乙醇( $99.5 \%)$ 购自国药集团化学试剂有限公
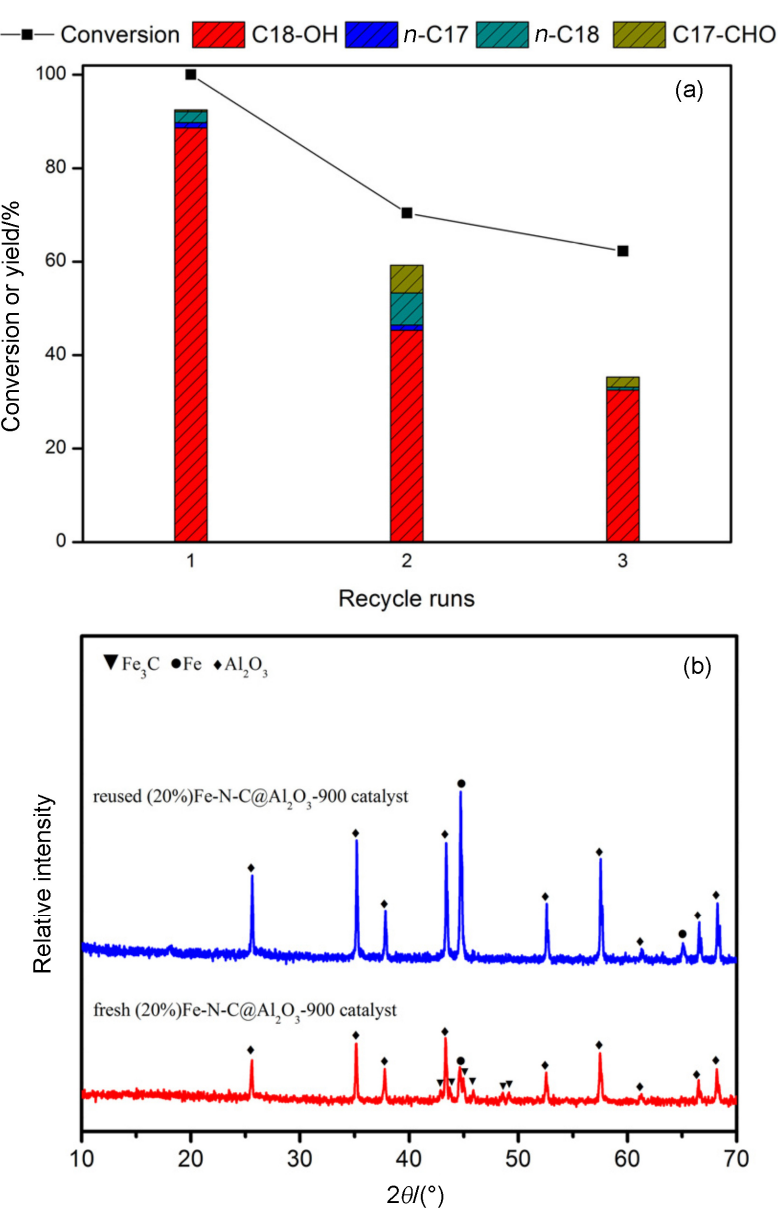

图 7 (a)铁催化剂稳定性实验以及(b)新鲜和重复使用的 $\mathrm{Fe}-\mathrm{N}-\mathrm{C} @, \mathrm{Al}_{2} \mathrm{O}_{3}-900$ 催化剂的 XRD 谱图

Figure 7 (a) Stability test of iron catalysts, and (b) XRD patterns of fresh and reused Fe-N-C @ $\mathrm{Al}_{2} \mathrm{O}_{3}-900$ catalysts

司. 1,10-菲罗啉一水合物 $(99 \%)$ 、三聚氰胺 $(98 \%) 、$ 活性 炭 $(99 \%) 、$ 、八烷 $(98 \%) 、$ 、八烯 $(90 \%)$ 、十八醇 $(98 \%)$ 及十八醛(95\%)购自 TCI 公司.

\section{2 催化剂制备}

铁催化剂是由铁和氮前驱体在各种载体上同时热 解制备的. 除非特殊说明, 否则铁的负载量(质量分数) 为 $20 \%$. 典型的制备方法如下：在室温下，在 $100 \mathrm{~mL}$ 乙 醇中首先加入一定量的乙酰丙酮铁. 待前体完全溶解 后, 向溶液中加入三聚氰胺. 然后将 $1 \mathrm{~g}$ 氧化铝添加到 混合物中, 并在 $60{ }^{\circ} \mathrm{C}$ 下将溶液搅拌 $16 \mathrm{~h}$, 然后在 $40{ }^{\circ} \mathrm{C}$ 下蒸发以去除乙醇溶剂. 将所得固体在 $60{ }^{\circ} \mathrm{C}$ 下干燥过 夜. 然后将所得混合物磨成细粉末, 在氩流量为 100 $\mathrm{mL} \cdot \mathrm{min}^{-1}$ 的管式炉中进行热解. 最终获得的催化剂记 为 $x \% \mathrm{Fe}-\mathrm{N}-\mathrm{C} @ \mathrm{~S}-\mathrm{T}$, 其中 $x \%$ 表示铁的负载量(质量分 数), $\mathrm{S}$ 表示载体类型, $\mathrm{T}$ 则表示热解温度. 为了制备具有 不同铁负载量的铁催化剂, 对三聚氰胺的量相应地进行 了调整使得铁/氮比保持不变. 我们发现不同批次制备 
的铁催化剂具有相同的催化性能，表明本工作报道的制 备方法具有较高的重复性.

\section{3 实验步骤}

羧酸的加氢反应均在购买于安徽科幂机械技术有 限公司的 $50 \mathrm{~mL}$ 反应釜中进行. 典型的操作步骤如下: 将 $0.5 \mathrm{mmol}$ 硬脂酸、 $100 \mathrm{mg}$ 非均相铁催化剂、 $0.5 \mathrm{mmol}$ 二十烷(内标)和 $20 \mathrm{~mL}$ 十二烷溶剂装入反应釜中的石英 内衬(用于消除釜金属内壁的影响)中. 反应釜用氢气吹 扫 3 次后在室温下充入需要的氢气. 反应在设定的温度 下进行 $0.5 \mathrm{~h}$ 后, 通过反应釜自带的取样装置取样后使 用气相色谱 $(\mathrm{GC})$ 分析产物, 色谱为 HP-5ms 柱(30 $\mathrm{m} \times$ $0.25 \mathrm{~mm} \times 0.25 \mu \mathrm{m}$ 毛细管柱). 为了确保反应的重现性, 一些反应至少重复了两次. 碳损失可归因于生成气相色 谱无法检测产物或结焦产物.

辅助材料(Supporting Information) 铁催化硬脂酸加 氢反应的气相色谱图. 这些材料可以免费从本刊网站 (http://sioc-journal.cn/)上下载.

\section{References}

[1] Huber, G. W.; Iborra, S.; Corma, A. Chem. Rev. 2006, 106, 4044

[2] Corma, A.; Iborra, S.; Velty, A. Chem. Rev. 2007, 107, 2411.

[3] Yang, Z.; Fu, Y.; Guo, Q. Chin. J. Org. Chem. 2015, 35, 273 (in Chinese).

(杨珍，傅尧，郭庆祥，有机化学, 2015, 35, 273.)

[4] Jamil, F.; Al-Haj, L.; Al-Muhtaseb, A. H.; Al-Hinai, M. A.; Baawain, M.; Rashid, U.; Ahmad, M. N. M. Rev. Chem. Eng. 2018, 34, 267.

[5] Besson, M.; Gallezot, P.; Pinel, C. Chem. Rev. 2014, 114, 1827.

[6] Gupta, S. Frost and Sullivan Market Insight 2004, 11075449.

[7] Adkins, H.; Folkers, K. J. Am. Chem. Soc. 1931, 53, 1095.

[8] Toba, M.; Tanaka, S.-I.; Niwa, S.-I.; Mizukami, F.; Koppány, Z.; Guczi, L.; Cheah, K.-Y.; Tang, T.-S. Appl. Catal., A 1999, 189, 243.

[9] Mendes, M.; Santos, O.; Jordao, E.; Silva, A. Appl. Catal., A 2001,
217, 253.

[10] Takeda, Y.; Nakagawa, Y.; Tomishige, K. Catal. Sci. Technol. 2012, 2, 2221

[11] Takeda, Y.; Tamura, M.; Nakagawa, Y.; Okumura, K.; Tomishige, K. ACS Catal. 2015, 5, 7034 .

[12] Ullrich, J.; Breit, B. ACS Catal. 2018, 8, 785.

[13] Manyar, H. G.; Paun, C.; Pilus, R.; Rooney, D. W.; Thompson, J. M.; Hardacre, C. Chem. Commun. 2010, 46, 6279.

[14] Rozmysłowicz, B.; Kirilin, A.; Aho, A.; Manyar, H.; Hardacre, C.; Wärnå, J.; Salmi, T.; Murzin, D. Y. J. Catalysis 2015, 328, 197.

[15] Toyao, T.; Siddiki, S. M.; Touchy, A. S.; Onodera, W.; Kon, K.; Morita, Y.; Kamachi, T.; Yoshizawa, K.; Shimizu, K. I. Chem.-Eur. J. 2017, 23, 1001.

[16] Kandel, K.; Chaudhary, U.; Nelson, N. C.; Slowing, I. I. ACS Catal. 2015, 5, 6719 .

[17] Wu, L.; Li, L.; Li, B.; Zhao, C. Chem. Commun. 2017, 53, 6152.

[18] Onyestyák, G.; Harnos, S.; Kalló, D. Catal. Commun. 2011, 16, 184.

[19] Gao, X.; Tong, D.; Zhong, H.; Jin, B.; Jin, F.; Zhang, H. RSC Adv. 2016, 6, 27623.

[20] Kong, X.; Fang, Z.; Bao, X.; Wang, Z.; Mao, S.; Wang, Y. J. Catalysis 2018, 367, 139.

[21] Jia, W.; Xu, G.; Liu, X.; Zhou, F.; Ma, H.; Zhang, Y.; Fu, Y. Energy Fuels 2018, 32, 8438.

[22] Song, S.; Wang, D.; Di, L.; Wang, C.; Dai, W.; Wu, G.; Guan, N.; Li, L. Chin. J. Catal. 2018, 39, 250

[23] Li, J.; Zhang, J.; Wang, S.; Xu, G.; Wang, H.; Vlachos, D. G. ACS Catal. 2019, 9, 1564

[24] Jagadeesh, R. V.; Surkus, A.; Junge, H.; Pohl, M.; Radnik, J.; Rabeah, J.; Huan, H.; Schunemann, V.; Bruckner, A.; Beller, M. Science 2013, 342, 1073.

[25] Cui, X.; Li, Y.; Bachmann, S.; Scalone, M.; Surkus, A.; Junge, K.; Topf, C.; Beller, M. J. Am. Chem. Soc. 2015, 137, 10652.

[26] Natte, K.; Neumann, H.; Jagadeesh, R. V.; Beller, M. Nat. Commun. 2017, 8, 1344.

[27] Li, J.; Liu, J.; Zhou, H.; Fu, Y. ChemSusChem 2016, 9, 1339.

[28] Li, J.; Liu, J.; Liu, H.; Xu, G.; Zhang, J.; Liu, J.; Zhou, G.; Li, Q.; $\mathrm{Xu}, \mathrm{Z}$; Fu, Y. ChemSusChem 2017, 10, 1436.

[29] Li, J.; Zhang, J.; Liu, H.; Liu, J.; Xu, G.; Liu, J.; Sun, H.; Fu, Y. ChemistrySelect 2017, 2, 11062.

[30] Li, J.; Sun, H.; Liu, J. X.; Zhang, J. J.; Li, Z. X.; Fu, Y. Mol. Catal. 2018, 452, 36.

[31] Leveneur, J.; Waterhouse, G. I. N.; Kennedy, J.; Metson, J. B.; Mitchell, D. R. G. J. Phys. Chem. C 2011, 115, 20978. 